\title{
Two Novel Variants in DYRK1B causative of AOMS3: Expanding the Clinical Spectrum
}

\section{Elvia Cristina Mendoza-Caamal}

National Institute of Genomic Medicine: Instituto Nacional de Medicina Genomica https://orcid.org/0000-0002-6224-6829

Francisco Barajas-Olmos

National Institute of Genomic Medicine: Instituto Nacional de Medicina Genomica

Elaheh Mirzaeicheshmeh

National Institute of Genomic Medicine: Instituto Nacional de Medicina Genomica

Ian Ilizaliturri-Flores

National Polytechnic Institute: Instituto Politecnico Nacional

\section{Carlos Aguilar-Salinas}

Salvador Zubiran National Institute of Medical Sciences and Nutrition: Instituto Nacional de Ciencias Medicas y Nutricion Salvador Zubiran

Donaji Gómez-Velasco

Salvador Zubiran National Institute of Medical Sciences and Nutrition: Instituto Nacional de Ciencias Medicas y Nutricion Salvador Zubiran Isabel Cicerón-Arellano

national institute of genomic medicine

Adriana Reséndiz-Rodríguez

National Institute of Genomic Medicine: Instituto Nacional de Medicina Genomica

Angélica Martínez-Hemández

National Institute of Genomic Medicine

Cecilia Contreras-Cubas

National Institute of genomic medicine

Sergio Islas-Andrade

National Institute of Genomic Medicine: Instituto Nacional de Medicina Genomica

Carlos Zerrweck

General Hospital Tlahuác

Humberto García-Ortiz

National Institute of Genomic Medicine

Lorena Orozco ( $\nabla$ lorozco@inmegen.gob.mx)

National Institute of Genomic Medicine https://orcid.org/0000-0002-5801-9180

\section{Research}

Keywords: AOMS3, diabetes, DYRK1B, metabolic syndrome, monogenic and obesity

Posted Date: December 23rd, 2020

DOI: https://doi.org/10.21203/rs.3.rs-133650/v1

License: (a) (i) This work is licensed under a Creative Commons Attribution 4.0 International License. Read Full License 


\section{Abstract}

Background: We investigated pathogenic DYRK1B variants causative of abdominal obesity-metabolic syndrome 3 (AOMS3) in a group of patients originally diagnosed with type 2 diabetes. All DYRK1B exons were analyzed in a sample of 509 unrelated adults with type 2 diabetes and 459 controls, all belonging to the DMS1 SIGMA-cohort (EXAC). We performed in silico analysis on missense variants using Variant Effect Predictor software. To evaluate co-segregation, predicted pathogenic variants were genotyped in other family members. We performed molecular dynamics analysis for the co-segregating variants.

Results: After filtering, Mendelian genotypes were confirmed in two probands bearing two novel variants, p.Arg252His and p.Lys68GIn. Both variants cosegregated with the AOMS3 phenotype in classic dominant autosomal inheritance with full penetrance. In silico analysis revealed protein affectation induced by both variants. For the first time, we describe age-dependent variable expressivity of this entity, with central obesity and insulin resistance apparent in childhood; morbid obesity, severe hypertriglyceridemia, and labile type 2 diabetes appearing before 40 years of age; and hypertension emerging in the fifth decade of life. We also report the two youngest individuals suffering from AOMS3.

Conclusions: Monogenic forms of metabolic diseases could be misdiagnosed and should be suspected in families with several affected members and earlyonset metabolic phenotypes that are difficult to control. Early diagnostic strategies and medical interventions, even before symptoms or complications appear, could be useful.

\section{Background}

Abdominal obesity-metabolic syndrome 3 (AOMS3 [MIM:615812]) is a rare autosomal dominant disorder caused by pathogenic variants in the dual-specificity tyrosine phosphorylation-regulated kinase 1B gene (DYRK1B) located on chromosome 19q13.2 (1). This monogenic form of metabolic syndrome (MetS) is characterized by abdominal obesity, type 2 diabetes, hypertension, and early-onset coronary artery disease (2). DYRK1B inhibits the Sonic Hedgehog and WNT pathways, increasing the expression of master adipogenic transcription factors CCAAT/enhancer binding protein (C/EBPalpha) and peroxisome proliferatoractivated receptor gamma (PPARgamma)(2). Moreover, DYRK1B induces the expression of glucose-6-phosphatase, a key enzyme in hepatic gluconeogenesis (3).

The identification of carriers of pathogenic variants in genes such as DYRK1B could be useful for establishing early diagnostic strategies and medical interventions in a reasonable number of affected individuals even before symptoms or complications appear. Until now, only two different mutations (p.Arg102Cys and p.His90Pro) in DYRK1B have been described as being causative of AOMS3 in three Iranian families and five unrelated Caucasian individuals (2). However, without a direct search for the causative variant, it could be possible that families with rare monogenic forms of metabolic diseases for unnoticed or are confused with multifactorial diseases, mainly in populations with a high prevalence of these entities. Therefore, we searched for DYRK1B variants in the exome sequencing data derived from 968 unrelated individuals (509 with type 2 diabetes) belonging to the DMS1 SIGMA-cohort (ExAC) (4), focusing on variants classified by Variant Effect Predictor (VEP) as deleterious and damaging to confirm their co-segregation with AOMS3. Here, we describe two novel DYRK1B mutations as causative of AOMS3 in two families previously misdiagnosed with type 2 diabetes.

\section{Results}

\section{Identification and co-segregation of pathogenic variants in the DYRK1B gene}

Of the 968 unrelated individuals, 52.6\% ( $n=509)$ had type 2 diabetes according to the American Diabetes Association criteria (5). The remaining 459 individuals were healthy subjects $>45$ years old with fasting glucose levels $<100 \mathrm{mg} / \mathrm{dL}$. We were able to identify 29 variants in $D Y R K 1 B$ (Supplemental Table S1), including seven missense variants and four variants predicted to be deleterious or damaging by VEP (Table 1). Two of these latter variants, p.Leu28Pro and p.Asp436Asn, were found in six heterozygote individuals ( $n=5$ and $n=1$, respectively), all of them without type 2 diabetes or clinical characteristics of AOMS3. The remaining two missense variants, p.Arg252His and p.Lys68Gln, were found in two unrelated individuals manifesting symptoms suggestive of AOMS3, such as childhood-onset abdominal obesity, type 2 diabetes, hypertriglyceridemia, and arterial hypertension. Both of these individuals also had a family history of obesity and type 2 diabetes, as well as premature death secondary to myocardial infarction in first and second-degree relatives. We were able to recruit three generations of these two families with 26 traceable members, 9 of whom were DYRK1B pathogenic-variant carriers ( 5 harbored the p.Arg252His variant and 4 p.Lys68GIn). The pedigree analysis revealed co-segregation of the DYRK1B genotype with the AOMS3 phenotype, showing a characteristic autosomal dominant inheritance pattern with full penetrance (Fig. 1) and age-dependent variable expressivity. None of the non-carrier family members had type 2 diabetes or previous diagnosis of dyslipidemia, and only one had arterial hypertension. The carriers had severe metabolic compromise characteristic of AOMS3 (Table 2), and we observed a significant difference between carriers and non-carriers of pathogenic variants; carriers had higher body mass index (BMI), hip/waist ratio, and fasting glucose (FG) and triglyceride levels. Furthermore, abdominal obesity started in childhood and progressed to morbid obesity in youth. The current mean BMI was $36.6 \mathrm{Kg} / \mathrm{m}^{2}$, but all patients had a higher BMI (mean $45.5 \mathrm{~kg} / \mathrm{m}^{2}$ ) before the diagnosis of type 2 diabetes. HOMA-IR and the Matsuda index (normal > 3) were impaired in all carriers, even in a child with normoglycemia (Supplemental Figure S1). Similarly, the mean age of diagnosis of type 2 diabetes was 34 years old, and most of them had difficult to control disease (mean HbA1c: $87 \mathrm{mmol} / \mathrm{mol}$ (10.1\%) and mean FG: $191.1 \mathrm{mg} / \mathrm{dL}$ ). The mean serum triglyceride levels were $315.9 \mathrm{mg} / \mathrm{dL}$, though all adult carriers underwent pharmacological treatment for dyslipidemia, except individual II.14 in family 2 , who had serum triglyceride levels of $650 \mathrm{mg} / \mathrm{dL}$. 
Table 1

Variant Effect Predictor analysis of missense variants in DYRK1B

\begin{tabular}{|lllll|}
\hline Variant & Nucleotide change & Amino acid change & SIFT $^{\mathrm{a}}$ & PolyPhen2 \\
\hline rs746933234 & c.755G $>$ A & p.Arg252His & Deleterious (0.02) & Probably damaging (0.996) \\
rs373850179 & c.202A $>$ C & p.Lys68GIn & Deleterious (0.01) & Possibly damaging (0.713) \\
\hline rs34587974 & c.83T $>$ C & p.Leu28Pro & Deleterious (0.01) & Possibly damaging (0.563) \\
\hline rs752428936 & c.1306G > A & p.Asp436Asn & Deleterious (0.05) & Probably damaging (0.957) \\
\hline rs148788670 & c.1733C $>$ T & p.Pro578Leu & Tolerated-low confidence (0.23) & Benign (0) \\
\hline rs144370928 & c.209G $>$ A & p.Arg70GIn & Tolerated (0.06) & Benign (0.047) \\
\hline rs771417583 & c.1666A $>$ C & p.Thr556Pro & Deleterious-low confidence (0) & Benign (0) \\
\hline aSIFT, Sorting Tolerant From Intolerant & & & \\
\hline
\end{tabular}


Table 2

Clinical characteristics of the 26 individuals genotyped in two families.

\begin{tabular}{|c|c|c|c|c|c|c|c|c|c|c|c|c|c|}
\hline Family/Mutation & & ID & Gender & $\begin{array}{l}\text { Age } \\
\text { years }\end{array}$ & $\begin{array}{l}\text { OB/Age } \\
\text { of onset }\end{array}$ & $\begin{array}{l}\mathrm{BMI} \\
\mathrm{Kg} / \mathrm{m}^{2}\end{array}$ & $\begin{array}{l}\text { BMI } \\
\text { befo- } \\
\text { re } \\
\text { T2Dt }\end{array}$ & $\begin{array}{l}\text { T2D } \\
\text { /Age of } \\
\text { diagno- } \\
\text { sis } \\
\text { years }\end{array}$ & $\begin{array}{l}\mathrm{FG} \\
\mathrm{mg} / \mathrm{dL}\end{array}$ & $\begin{array}{l}\mathrm{HbA} 1 \mathrm{c} \\
\mathrm{mmol} / \mathrm{mol}\end{array}$ & $\begin{array}{l}\text { HbA1c } \\
\%\end{array}$ & $\begin{array}{l}\text { Dyslipi- } \\
\text { demia } \\
\text { /Age } \\
\text { of } \\
\text { onset } \\
\text { years }\end{array}$ & $\begin{array}{l}\text { TG } \\
\mathrm{mg}\end{array}$ \\
\hline \multirow[t]{12}{*}{ F1/p.Arg252His } & \multirow{5}{*}{$\begin{array}{l}\text { No- } \\
\text { carries }\end{array}$} & IV.6 & M & 12 & No & 24 & NA & No & 89 & & - & No & 145 \\
\hline & & IV.4 & $M$ & 13 & No & 19.7 & NA & No & 83 & 34 & 5.3 & No & 86 \\
\hline & & IV.2 & $\mathrm{F}$ & 18 & No & 26.4 & NA & No & 83 & 34 & 5.3 & No & 109 \\
\hline & & III.7 & M & 42 & Yes/42 & 31.6 & NA & No & 68 & & - & No & 118 \\
\hline & & III.6 & $\mathrm{F}$ & 41 & Yes/41 & 32.5 & NA & No & 67 & 37 & 5.5 & No & 83 \\
\hline & \multirow[t]{5}{*}{ Carries } & IV.5 & M & 11 & Yes/Childhood & 30 & NA & No & 98 & 37 & 5.5 & No & 130 \\
\hline & & III.10 & $\mathrm{F}$ & 34 & Yes/Childhood & 35.6 & 35.6 & Yes/34 & 168 & 76 & 9.1 & Yes/29 & 479 \\
\hline & & III.8 & $\mathrm{F}$ & 45 & Yes/Childhood & 36.7 & 42.3 & Yes/43 & 165 & 61 & 7.7 & Yes/44 & 412 \\
\hline & & III.5 & M & 46 & Yes/Childhood & 41.5 & 47.8 & Yes/41 & 120 & 51 & 6.8 & Yes/30 & 341 \\
\hline & & II.5 & $\mathrm{F}$ & 69 & Yes/Childhood & 33.8 & 35.7 & Yes/41 & 124 & 74 & 8.9 & Yes/62 & 249 \\
\hline & \multirow{2}{*}{$\begin{array}{l}\text { Average } \\
\pm \text { SD } \\
\text { carriers } \\
\text { with } \\
\text { T2D }\end{array}$} & - & - & $48.5 \pm$ & \multirow[t]{2}{*}{-} & \multirow{2}{*}{$\begin{array}{l}36.9 \pm \\
3.2\end{array}$} & 40.3 & 39.7 & \multirow{2}{*}{$\begin{array}{l}144.2 \\
\pm \\
25.7 *\end{array}$} & \multirow[t]{2}{*}{$65 \pm 11^{\star}$} & $8.1 \pm$ & $41.2 \pm$ & 370 \\
\hline & & & & 14.7 & & & $\begin{array}{l} \pm 5.8 \\
*\end{array}$ & \pm 3.9 & & & $\begin{array}{l}1.0 \\
*\end{array}$ & 15.4 & 98.5 \\
\hline \multirow[t]{17}{*}{ F2/p.Lys68GIn } & \multirow{12}{*}{$\begin{array}{l}\text { No- } \\
\text { carries }\end{array}$} & III.21 & $M$ & 11 & No & 18.6 & NA & No & 86 & & - & No & 48 \\
\hline & & III.5 & $\mathrm{F}$ & 12 & No & 16.9 & NA & No & 75 & & - & No & 75 \\
\hline & & III.20 & $\mathrm{F}$ & 14 & No & 25.4 & NA & No & 88 & & - & No & 83 \\
\hline & & III.12 & M & 17 & Yes/17 & 37 & NA & No & 90 & & - & No & 106 \\
\hline & & III. 2 & $\mathrm{~F}$ & 22 & No & 21.5 & NA & No & 82 & & - & No & 59 \\
\hline & & III.1 & $\mathrm{F}$ & 27 & No & 29.5 & NA & No & 75 & & - & No & 87 \\
\hline & & III. 10 & M & 35 & No & 20.7 & NA & No & 81 & & - & No & 56 \\
\hline & & II. 15 & $\mathrm{~F}$ & 36 & No & 25.9 & NA & No & 94 & & - & No & 79 \\
\hline & & II.13 & $\mathrm{F}$ & 41 & No & 24.5 & NA & No & 77 & & - & No & 66 \\
\hline & & II. 2 & $\mathrm{~F}$ & 49 & Yes/44 & 38.8 & NA & No & 109 & 43 & 6.1 & No & 105 \\
\hline & & II.1 & $\mathrm{F}$ & 50 & Yes/50 & 31.4 & NA & No & 89 & & - & No & 85 \\
\hline & & 1.2 & $\mathrm{~F}$ & 70 & No & 20.7 & NA & No & 85 & & - & No & 75 \\
\hline & \multirow[t]{4}{*}{ Carries } & III.19 & M & 18 & No & 26.4 & NA & No & 102 & 41 & 5.9 & Yes/16 & 125 \\
\hline & & III.18 & $M$ & 27 & Yes/Childhood & 40.4 & 50.8 & Yes/18 & 276 & 113 & 12.5 & Yes/24 & 231 \\
\hline & & II.14 & M & 40 & Yes/Childhood & 51.9 & 55.6 & Yes/29 & 257 & 135 & 14.5 & Yes/37 & 650 \\
\hline & & II.12 & $\mathrm{F}$ & 49 & Yes/Childhood & 33.4 & 50.8 & Yes/31 & 228 & 99 & 11.2 & Yes/31 & 226 \\
\hline & $\begin{array}{l}\text { Average } \\
\pm \text { SD } \\
\text { carriers } \\
\text { with } \\
\text { T2D }\end{array}$ & - & - & $\begin{array}{l}38.7 \\
\pm 11\end{array}$ & - & $\begin{array}{l}41.9 \pm \\
9.3\end{array}$ & $\begin{array}{l}52.4 \pm \\
2.8 \\
*\end{array}$ & $26 \pm 7$ & $\begin{array}{l}253.6 \\
\pm \\
24.1^{*}\end{array}$ & $\begin{array}{l}97 \pm 40.1 \\
\text { * }\end{array}$ & $\begin{array}{l}12.7 \\
\pm 1.7 \\
*\end{array}$ & $\begin{array}{l}30.67 \\
\pm 6.51\end{array}$ & $\begin{array}{l}369 \\
\pm \\
243\end{array}$ \\
\hline
\end{tabular}

ID, identification; OB, obesity; BMI, body mass index; T2D, type 2 diabetes; FG, fasting glucose; HbA1c, glycosylated hemoglobin; $T$, triglyceride; Chol, total ch hypertension; SBP, systolic blood pressure; DBP, diastolic blood pressure; $M$, male; $F$, female; NA, not apply; + , referred value by the patient. * adjusted p-value $k$ p.Arg252His with T2D and the average value of p.Arg252His with T2D.

The clinical record documented that arterial hypertension was the last trait to become apparent, and it was present in 4 of 5 carriers older than 40 years old (mean age of the diagnosis of arterial hypertension was 47 years). The pulse wave velocity increased in all carriers, even in the absence of arterial hypertension. p.Lys68GIn carriers had a higher BMI and worse diabetes control than p.Arg252His carriers. Furthermore, all three p.Lys68GIn carriers, but not the p.Arg252His carriers, had an increased albumin/creatinine ratio (Supplemental Table S2).

However, we were able to document metabolic disorders in two carrier males in their second decade of life. The youngest was an 11-year-old child who carried the p.Arg252His variant (IV.5 in family 1), had obesity (BMI: $30 \mathrm{Kg} / \mathrm{m}^{2}$, weight > 95th percentile of CDC grow charts), and demonstrated insulin resistance 
(HOMA-IR: 5.44) but not other metabolic abnormalities. The second carrier was an 18-year-old who carried the p.Lys68GIn variant (III.19 in family 2), was overweight (BMI: $26.4 \mathrm{Kg} / \mathrm{m}^{2}$, weight 90-95th percentile of CDC grow charts), serum FG $102 \mathrm{mg} / \mathrm{dL}$, and hypertriglyceridemia since 16 years old; he was undergoing pharmacological treatment. Notably, both carriers had a strictly controlled diet because of the family history of type 2 diabetes.

\section{Molecular dynamics}

To gain more insight into the effect of the variants on protein structure and function, we performed molecular dynamics (MD) simulation trajectory analysis of DYRK1B-252Arg and DYRK1B-252His (Fig. 2). The root mean square deviation (RMSD) values indicate that DYRK1B-252His took a longer period of time to reach equilibration (Fig. 2A). The root mean square fluctuation (RMSF) analysis documented stability and structural changes in the $\mathrm{N}$ - and $\mathrm{C}$-terminal regions, possibly due to the lack of hydrogen bonds (Fig. 2B, brown and yellow boxes). In addition, analyses revealed that, in contrast, to the results when Arg was present at position 252, His252 lost the hydrogen bonds with Pro552 and Ser554, resulting in conformational changes in the catalytic site and potentially losing functionality (Fig. 2C - F).

Despite the DYRK1B-68GIn mutant showing no structural changes and relative stability, in silico analysis revealed a shortening of three amino acids (positions 65-68) within the nuclear localization sequence (positions 65-86), suggesting that, although its catalytic function could not be affected, it could accumulate in the cytosol.

\section{Discussion}

Over the past few years, there have been many attempts to gain more insight into the genetic factors involved in metabolic diseases (6-8). Multiple genetic variants have been shown to participate in the pathogenesis of each of the traits of metabolic syndrome. The polygenic nature of these conditions implies that the effect of the majority of genetic variants in these disorders is small (6). However, families in which autosomal dominant inheritance is present have been used to search for rare mutations in genes with a strong contribution $(9,10)$. The availability of exome sequencing is leading to the rapid identification of new players in the pathogenesis of metabolic diseases. This is the case for DYRK1B mutations, which cause a rare monogenic form of MetS known as AOMS3(2). This syndrome has been described as the presence of abdominal obesity, type 2 diabetes, hypertension, and early-onset coronary artery disease (2). Pathological DYRK1B variants result in the enhanced expression of transcription factors C/EBPalpha and PPARgamma, leading to increased adipogenesis. In addition, DYRK1B increases glucose-6-phosphatase, which is strongly associated with insulin resistance, explaining the metabolic phenotypes characterizing $\operatorname{AOMS} 3(2,3)$.

The participation of DYRK1B in MetS is poorly studied. Six years after DYRK1B was associated with AOMS3, only a few carriers have been reported (1). Furthermore, only two missense DYRK1B mutations (p.Arg102Cys and p.His90Pro) have been identified in these individuals (2). It is possible for rare diseases that mimic symptoms of common diseases to be confused with them. The prevalence of metabolic diseases in Mexico is one of the highest in the world (11, 12), and rare metabolic diseases are often hidden behind them; therefore, families with rare monogenic forms can remain unnoticed.

Next generation sequencing technologies have greatly improved the possibility of identifying rare pathogenic variants involved in monogenic diseases $(4,10)$ In this study, after analyzing the sequence of all DYRK1B exons in a sample of 968 adult, including 509 with type 2 diabetes (SIGMA-ExAC) (13), we found 29 variants. SIFT and PolyPhen predicted that four of them (p.Leu28Pro, p.Asp436Asn, p.Arg252His, and p.Lys68GIn) have a deleterious and damaging effect. The p.Leu28Pro variant was described previously as having a protective effect against type 2 diabetes in a phenome-wide association study (14). In this study, we found five heterozygotes individuals with neither type 2 diabetes nor AOMS3, but we were not able to recruit the familial relatives to confirm its protective effect. The p.Asp436Asn variant was found in a male heterozygote, who was 98 years old and metabolically healthy. However, p.Arg252His and p.Lys68GIn exhibited co-segregation with the AOMS3 phenotype with classic dominant autosomal inheritance and full penetrance. Both variants were absent in the 1000 Genomes database (15), but the ExAC Browser reports, in addition to those reported here, a South Asian individual heterozygous for p.Arg252His and a nonFinnish European carrier heterozygous for p.Lys68GIn (16). MD structural analyses predicted that, when DYRK1B-252His was present, the formation of three hydrogen bonds was impaired, with instability in the $\mathrm{N}$ - and C-terminal regions. In contrast, when DYRK1B-68GIn was present, the binding motif in the nuclear localization sequence was shortened without significant changes to the protein structure. The effect of these variants could be similar to those documenting that DYRK1B-102Cys and DYRK1B-90Pro variants cause changes to the structure and perinuclear aggregation but barely affect the kinase activity (17). We classified p.Arg252His and p.Lys68GIn as causative of AOMS3, in agreement with the American College of Medical Genetics and Genomics standards and guidelines (18).

Compared to non-carriers of pathogenic variants, we found that carriers had higher BMI, hip/waist ratio, and FG and triglyceride levels. Furthermore, pulse wave velocity was increased in all carriers, even in the absence of arterial hypertension, explaining the high cardiovascular risk found in this condition. Insulin action was decreased in all but one case, but the insulinogenic index was significantly decreased in all carriers, even in normoglycemic individuals, suggesting that the remarkable severity of hyperglycemia found in this condition results from a combination of moderate insulin resistance and a moderate to severe defect in insulin secretion.

Notably, we are describing additional features of the disease and manifestations that develop throughout life. Our findings exhibit age-dependent variance in expressivity in all patients, with some clinical features apparent at a very early age and other manifestations appearing later in life. Central obesity and insulin resistance started during childhood and then progressed rapidly to morbid obesity and labile type 2 diabetes. They also had severe hypertriglyceridemia with onset as a teenager. Similarly, AOMS3 patients developed hypertension in the fifth decade of life, and both families had a history of premature death due to cardiovascular events. Another interesting finding was that p.Lys68GIn carriers had a higher BMI, worse diabetes control and an increased albumin/creatinine ratio than the p.Arg252His carriers, suggesting an allelic heterogeneity. 


\section{Conclusions}

How can these findings improve public health? AOMS3 should be suspected in individuals with the clinical outcome described above and a family history of affected first degree relatives. Assessment of the first-degree relatives should be performed routinely in subjects with extreme obesity. An autosomal dominant inheritance pattern should be systematically sought. Identifying variants involved in rare disorders could bring to light new genes associated with common diseases and may have implications for screening and targeted therapy. Otherwise, cascade genetic screening increases the possibility of finding presymptomatic carriers and lead to the implementation of strategies to prevent or delay the disease course, benefiting a reasonable number of affected families. The large number of cases and remarkably large structure of the Mexican families are unique opportunities to identify new genetic variants in understudied populations. Special care should be taken with the youngest members of the families, in whom cardiovascular prevention should be implemented early in life.

\section{Material And Methods Study participants}

We included 968 unrelated adult Mexican Mestizos belonging to the DMS1 SIGMA-cohort (13) who were previously sequenced by Sure-Select Human All Exon v2.0 (Illumina) and included in the ExAC project (4). A peripheral blood sample was collected after fasting for at least 8 hours. The following clinical and biochemical data were obtained for all participants of the DMS1 cohort using the Synchron CX5 Analyzer System (Beckman Coulter Fullerton, CA, USA): FG $(\mathrm{mg} / \mathrm{dL}), \mathrm{HDL}(\mathrm{mg} / \mathrm{dL})$, and serum triglycerides (mg/dL). HbA1c levels were measured using the IN2it analyzer (Bio-Rad, Hercules, CA, USA). Blood pressure was measured using a digital blood pressure monitor (HEM-907XL, OMRON). Weight and height were measured using a body composition monitor (HBF-500 INT, OMRON) and electronic stadiometer (ADE Germany). Waist circumference was measured midway between the inferior margin of the ribs and the border of the iliac crest using a flexible clinical measuring tape.

The study was carried out according to the Declaration of Helsinki and was approved by the Research, Ethics, and Biosafety Human Committees of the Instituto Nacional de Medicina Genómica (INMEGEN) in Mexico City. All participants provided written informed consent. They were recruited from August 2017 to December 2018, all of them inhabited the Valley of Mexico.

\section{Identification of pathogenic variants}

We identified DYRK1B variants by analyzing the exon sequences in each individual. To find the deleterious and damaging variants, we annotated them using the VEP toolset (19). Missense variants that were predicted as deleterious or affecting the protein structure were used to perform genotype-phenotype linkages. Individuals identified as carriers of these variants and had clinical manifestations suggesting AOMS3 were re-contacted and invited, along with their family members, to participate in a familial co-segregation study. All individuals who participated in the family segregation study provided written informed consent; in the case of children, the parents provided written consent and the children assented.

Genotyping was performed by Sanger sequencing using specific primers: p.Arg252His forward primer, CCTTTCTTCTCTGGCCAT; p.Arg252His reverse primer, ACCCAAACTACTAGCCGTGC; p.Lys69GIn forward primer, TGCCAGCAGCCTTACAGTT; p.Lys69GIn reverse primer, CCACTGCGCAACGATGTAGTC. The obtained sequences were analyzed by 4Peaks V1.8 (20).

The same clinical, demographic, and biochemical data described for index cases were obtained for each family participant. In addition, seven carriers (four p.Arg252His and three p.Lys69GIn) and three non-carriers were clinically and biochemically re-evaluated at the Unidad de Investigación de Enfermedades Metabólicas, Instituto Nacional de Ciencias Médicas y Nutrición. The assessment included fasting biochemical measurements (i.e., clinical chemistry, liver panel, and lipid profile), estimation of body composition, pulse wave velocity measurements, and albumin/creatinine ratio in a spot urine sample. The FG concentration was measured by an automated glucose analyzer (Yellow Springs Instruments $\mathrm{Co}$.). The serum insulin concentration was measured by a chemiluminescent immunoassay (Beckman Coulter Access 2) and HbA1c levels by HPLC (Variant II Turbo, BIORAD). Cholesterol, triglycerides, HDL, apolipoprotein B, uric acid, creatinine, and hepatic enzymes levels were measured using colorimetric assays (Unicel DxC 600 Synchron Clinical System Beckman Coulter). LDL was calculated by the Friedewald equation when the triglyceride concentration was $<250 \mathrm{mg} / \mathrm{dL}$.

\section{Molecular dynamics}

The wild-type amino acid sequence of DYRK1B (Q9Y463) from the UniProt database (21) was modeled to obtain the 3D protein structure using the I-TASSER server (22). The structures of mutated proteins were predicted using the predicted wild-type protein and VMD v1.9.3 software (23). Next, we carried out an atomistic MD simulation with explicit atom representation for proteins, water, and ions under force-field using the CHARMM package and NAMD v2.3 software (24). Periodic boundary conditions, particle mesh Ewald, and a non-bonded cut-off of $14 \AA$ and 2 fs time step were used. The isothermal-isobaric conditions were maintained with a Langevin thermostat $(310 \mathrm{~K})$ and Langevin piston barostat ( $1 \mathrm{~atm})$. For each model, the system was subjected to energy minimization for 1000 steps, followed by equilibration for $5 \mathrm{~ns}$, and the simulation continued for 50 ns without restraints. In the simulations analyses, we used the VEGAZZ V3.1.2 (25), Carma (26), and R cran project V3.4 (27) programs. The MD analysis included the RMSD and the RMSF.

\section{Statistical analysis}

Clinical data are reported as mean \pm standard deviation. Data were analyzed using R cran project v3.4 (27) and a general linear model test for comparing metabolite levels between carriers and non-carriers. Gender- and age-adjusted $P<0.05$ was considered significant.

\section{Abbreviations}


Abdominal obesity-metabolic syndrome 3

DYRK1

$B$ dual-specificity tyrosine phosphorylation-regulated kinase $1 \mathrm{~B}$

C/EBPalpha

transcription factors CCAAT/enhancer binding protein

FG

fasting glucose

INMEGEN

Instituto Nacional de Medicina Genómica

MD

molecular dynamics

MetS

metabolic syndrome

PPARgamma

peroxisome proliferator-activated receptor gamma

VEP

Variant Effect Predictor

RMSD

root mean square deviation

RMSF

root mean square fluctuation

\section{Declarations}

\section{Ethics approval and consent to participate}

The study was carried out according to the Declaration of Helsinki and was approved by the Research, Ethics, and Biosafety Human Committees of the Instituto Nacional de Medicina Genómica (INMEGEN) in Mexico City.

\section{Consent for publication}

Not applicable

\section{Availability of data and materials}

The datasets generated and analysed during the current study are not publicly available due this work is part of a larger project but are available from the corresponding author on reasonable request.

\section{Competing interests}

The authors declare that they have no competing interests.

\section{Funding}

This study was partially supported by Instituto Carlos Slim de la Salud, A.C.

\section{Author's contributions}

E.C.M-C. and F.B-O. were involved in all aspects of the study and wrote the first draft of the manuscript. L.O. contributed to the study design, analysis, interpretation of data, and editing of the manuscript. E.M. and I.I-F. contributed to the molecular dynamics analysis and interpretation of data. C.A.A-S., D.V.GV., I.C-A., A.R-R., A.M-H., C.C-C., S.I-A., C.Z., and H.G-O. contributed to the study design, interpretation of data, and sample collection. All authors were involved in writing the paper and gave final approval of the submitted and published versions.

\section{Acknowledgments}

The authors would like to thank the staff of the Immunogenomics and Metabolic Disease Laboratory/INMEGEN and the Endocrinology and Metabolism Department/ Instituto Nacional de Ciencias Médicas y Nutrición for all their support, particularly Juan Luis Jiménez-Ruiz, María Guadalupe Salas-Martínez, Yolanda Saldaña-Alvarez, Luz Elizabeth Guillen-Pineda, María Del Carmen Moreno-Villatoro, and Adriana Cruz-Lopez. We are thankful to the study volunteers for all their work and support throughout the realization of the study.

\section{References}

1. OMIM Entry - \# 615812 - ABDOMINAL OBESITY-METABOLIC SYNDROME 3; AOMS3 [Internet]. [cited 2019 Apr 30]. Available from: http://omim.org/entry/615812. 
2. Keramati AR, Fathzadeh M, Go G-W, Singh R, Choi M, Faramarzi S, et al. A Form of the Metabolic Syndrome Associated with Mutations in. N Engl J Med. 2014 May;15:370:1909-19.

3. von Groote-Bidlingmaier F, Schmoll D, Orth HM, Joost HG, Becker W, Barthel A. DYRK1 is a co-activator of FKHR (FOXO1a)-dependent glucose-6phosphatase gene expression. Biochem Biophys Res Commun. 2003 Jan 17;300(3):764-9.

4. Lek M, Karczewski KJ, Minikel EV, Samocha KE, Banks E, Fennell T, et al. Analysis of protein-coding genetic variation in 60,706 humans. Nature. 2016 Aug 17;536:285.

5. Association AD. 2. Classification and Diagnosis of Diabetes: Standards of Medical Care in Diabetes-2019. Diabetes Care. 2019 Jan 1;42(Supplement 1):S13-28.

6. Zafar U, Khaliq S, Ahmad HU, Manzoor S, Lone KP. Metabolic syndrome: an update on diagnostic criteria, pathogenesis, and genetic links. Horm Athens Greece. 2018 Sep;17(3):299-313.

7. Saklayen MG. The Global Epidemic of the Metabolic Syndrome. Curr Hypertens Rep. 2018 Feb;26(2):12. 20 (.

8. Sherling DH, Perumareddi P, Hennekens CH. Metabolic Syndrome: Clinical and Policy Implications of the New Silent Killer. J Cardiovasc Pharmacol Ther. 2017 Jul 1;22(4):365-7.

9. Serra-Juhé C, Martos-Moreno G, Bou de Pieri F, Flores R, Chowen JA, Pérez-Jurado LA, et al. Heterozygous rare genetic variants in non-syndromic earlyonset obesity. Int J Obes 2005. 2020;44(4):830-41.

10. DiStefano JK, Kingsley CB. Identification of Disease Susceptibility Alleles in the Next Generation Sequencing Era. Methods Mol Biol Clifton NJ. 2018;1706:3-16.

11. Rojas R, Aguilar-Salinas CA, Jiménez-Corona A, Shamah-Levy T, Rauda J, Avila-Burgos L, et al. Metabolic syndrome in Mexican adults: results from the National Health and Nutrition Survey 2006. Salud Pública México. 2010;52(Suppl 1):11-8.

12. Gutiérrez-Solis AL, Datta Banik S, Méndez-González RM. Prevalence of Metabolic Syndrome in Mexico: A Systematic Review and Meta-Analysis. Metab Syndr Relat Disord. 2018;16(8):395-405.

13. SIGMA Type 2 Diabetes Consortium. Williams AL, Jacobs SBR, Moreno-Macías H, Huerta-Chagoya A, Churchhouse C, et al. Sequence variants in SLC16A11 are a common risk factor for type 2 diabetes in Mexico. Nature. 2014 Feb 6;506(7486):97-101.

14. Mirshahi T, Murray MF, Carey DJ. The metabolic syndrome and DYRK1B. N Engl J Med. 2014 Aug;21(8):784-5. 371(.

15. 1000 Genomes. | A Deep Catalog of Human Genetic Variation [Internet]. [cited 2019 Nov 19]. Available from: https://www.internationalgenome.org/.

16. ExAC Browser [Internet]. [cited 2019 Nov 19]. Available from: http://exac.broadinstitute.org/.

17. Jhaisha SA, Widowati EW, Kii I, Sonamoto R, Knapp S, Papadopoulos C, et al. DYRK1B mutations associated with metabolic syndrome impair the chaperone-dependent maturation of the kinase domain. Sci Rep. 2017 Jul;25(1):6420. 7 (.

18. Richards S, Aziz N, Bale S, Bick D, Das S, Gastier-Foster J, et al. Standards and guidelines for the interpretation of sequence variants: a joint consensus recommendation of the American College of Medical Genetics and Genomics and the Association for Molecular Pathology. Genet Med Off $\mathrm{J}$ Am Coll Med Genet. 2015 May;17(5):405-24.

19. McLaren W, Gil L, Hunt SE, Riat HS, Ritchie GRS, Thormann A, et al. The Ensembl Variant Effect Predictor. Genome Biol. 2016 Dec;17(1):122.

20. 4Peaks. For peaks, four peaks. The DNA sequence trace viewer for OS X [Internet]. [cited 2019 Nov 19]. Available from:

https://nucleobytes.com/4peaks/index.html.

21. UniProt [Internet]. [cited 2019 Nov 19]. Available from: https://www.uniprot.org/.

22. Yang J, Yan R, Roy A, Xu D, Poisson J, Zhang Y. The I-TASSER Suite: protein structure and function prediction. Nat Methods. 2015 Jan;12(1):7-8.

23. Humphrey W, Dalke A, Schulten K. VMD: Visual molecular dynamics. J Mol Graph. 1996 Feb 1;14(1):33-8.

24. Phillips JC, Braun R, Wang W, Gumbart J, Tajkhorshid E, Villa E, et al. Scalable molecular dynamics with NAMD. J Comput Chem. 2005 Dec;26(16):1781802.

25. Pedretti A, Villa L, Vistoli G. VEGA: a versatile program to convert, handle and visualize molecular structure on Windows-based PCs. J Mol Graph Model. 2002 Aug;21(1):47-9.

26. Glykos NM. Software news and updates. Carma: a molecular dynamics analysis program. J Comput Chem. 2006 Nov 15;27(14):1765-8.

27. R: The R Project for Statistical Computing [Internet]. [cited 2019 Nov 19]. Available from: https://www.r-project.org/.

\section{Figures}




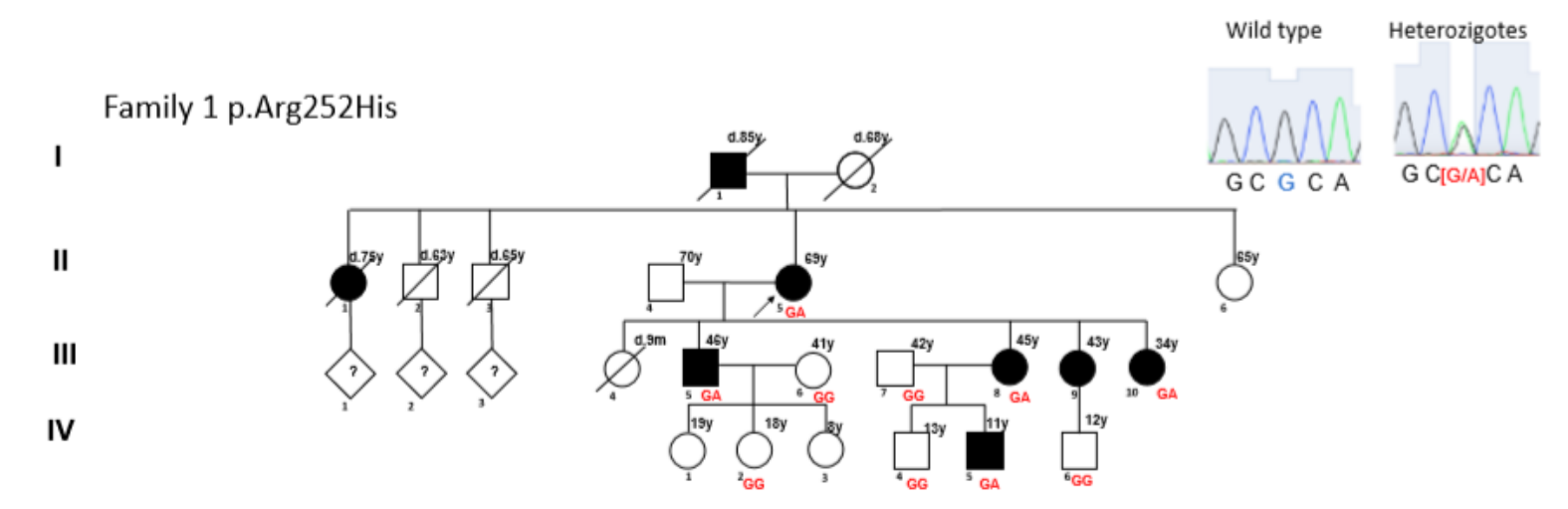

Family 2 p.Lys68Gln

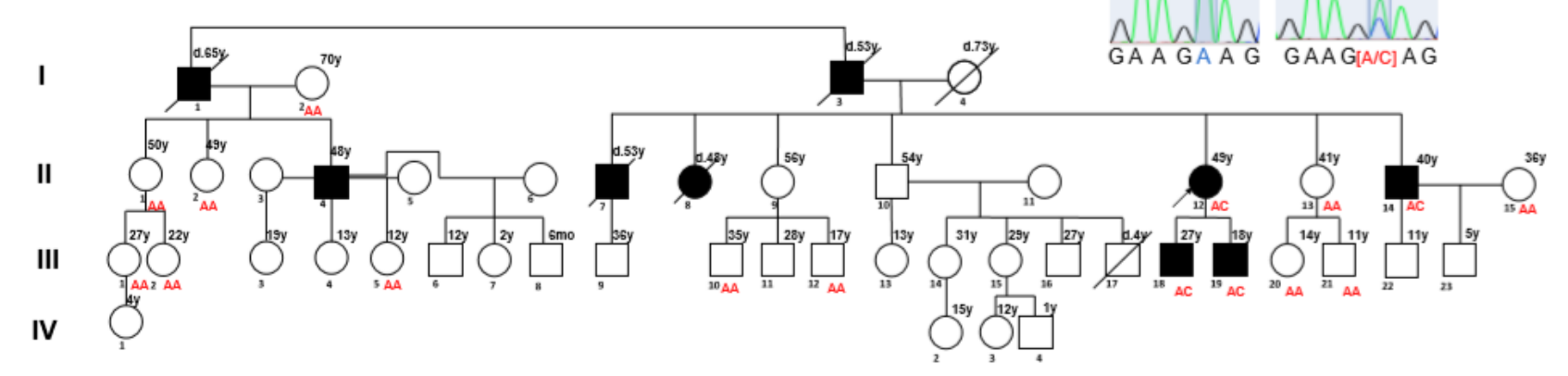

Figure 1

Pedigree of two families with an autosomal dominant inheritance pattern of DYRK1B mutations associated with AOMS3. Family 1 with p.Arg252His (Arg: CGC/His: CAC). Family 2 showing the p.Lys68GIn mutation (Lys: AAG/GIn: CAG). The index cases (II.5 in family 1 and II.12 in family 2) are indicated by arrows. Genotypes are presented under each individual. Family members with clinical signs compatible with AOMS3 are indicated by solid symbols. Slashes indicate that the individual is deceased. 
A)

B)
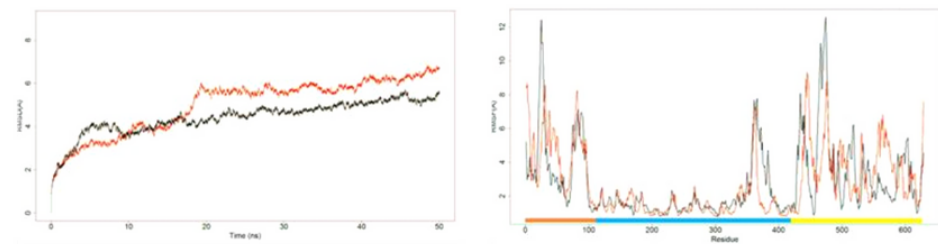

C)

D)
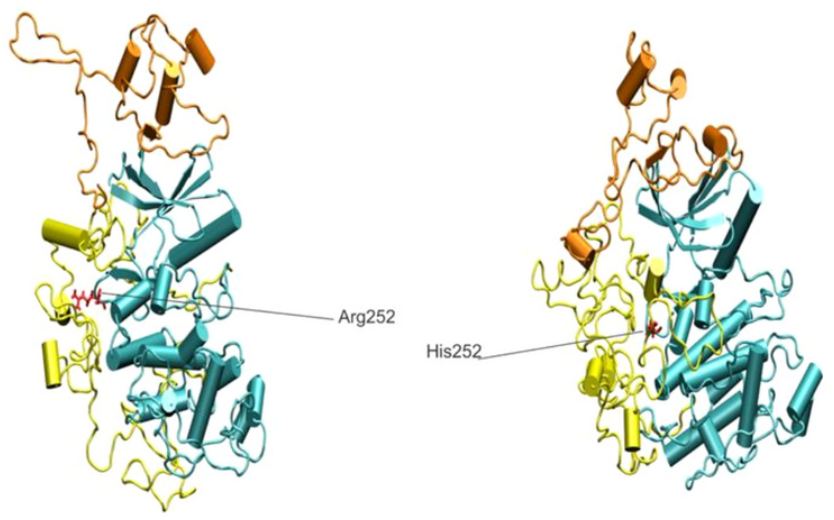

E)

F)
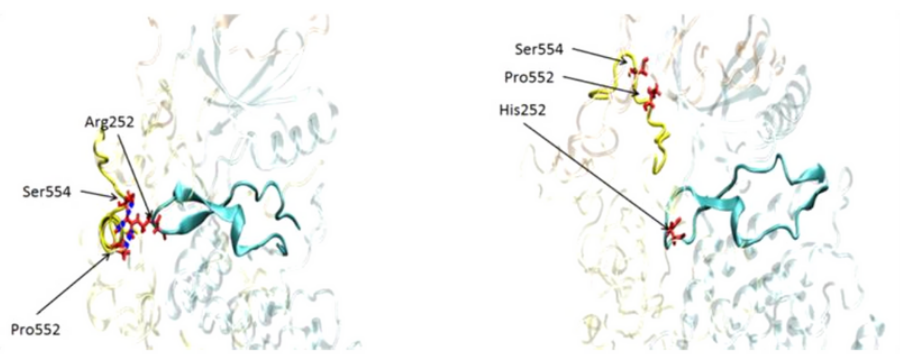

\section{Figure 2}

Molecular dynamic analysis of DYRK1B-252His. A) Root mean square deviation (RMSD) of atomic positions, analysis of wild-type protein (black line), and DYR1B-252His (red line). B) Root mean square fluctuation (RMSF) of the wild-type protein (black line) and DYR1B-252His (red line). C) Wild-type structure. D) Structure of DYRK1B-252His. The protein structure colors are the same as previously described. E) Region of interest zoomed-in for Arg252. The hydrogen bonds are depicted by the blue dots. F) Same region of interest with the His252 variant.

\section{Supplementary Files}

This is a list of supplementary files associated with this preprint. Click to download.

- Supplementalmaterialfiles.docx 